\title{
Clinical Utility of an Intimate Partner Violence Screening Tool for Female VHA Patients
}

\author{
Katherine M. Iverson, $P h D^{1,2}$, Matthew W. King, PhD ${ }^{7}$, Patricia A. Resick, PhD 1,2,3, \\ Megan R. Gerber, MD, MPH $H^{4,5}$, Rachel Kimerling, $P h D^{6,7}$, and Dawne Vogt, PhD ${ }^{1,2}$
}

\begin{abstract}
'Women's Health Sciences Division of the National Center for PTSD, VA Boston Healthcare System, Boston, MA, USA; ${ }^{2}$ Department of Psychiatry, Boston University School of Medicine, Boston, MA, USA; ${ }^{3}$ Department of Psychology, Boston University, Boston, MA, USA; ${ }^{4}$ Women's Health, VA Boston Healthcare System, Boston, MA, USA; ${ }^{5}$ Department of General Internal Medicine, Boston University School of Medicine, Boston, MA, USA; ${ }^{6}$ National Center for PTSD, VA Palo Alto Health Care System, Menlo Park, CA, USA; ${ }^{7}$ Center for Health Care Evaluation, VA Palo Alto Health Care System, Menlo Park, CA, USA.
\end{abstract}

OBJECTIVES: Female Veterans are at high risk for physical, sexual, and psychological forms of intimate partner violence (IPV) victimization. This study evaluated the accuracy of a brief IPV victimization screening tool for use with female Veterans Health Administration (VHA) patients.

DESIGN: Participants completed a paper-and-pencil mail survey that included the four-item Hurt/Insult/ Threaten/Scream (HITS) and the 39-item Revised Conflict Tactics Scales (CTS-2). Operating characteristics, including sensitivity and specificity, were calculated using the CTS-2 as the reference standard for pastyear IPV.

PARTICIPANTS: Female veterans from a roster of randomly selected female patients of the New England VA Healthcare System. Women must have reported being in an intimate relationship in the past year to be included.

MAIN MEASURES: Primary measures included the HITS (index test) and the CTS-2 (reference standard).

KEY RESULTS: This study included 160 women. The percentage of women who reported past-year IPV, as measured by any physical assault, sexual coercion, and/or severe psychological aggression on the CTS-2, was $28.8 \%$. The receiver-operator characteristic curve demonstrated that the HITS cutoff score of 6 maximizes the true positives while minimizing the false positives in this sample. The sensitivity of the optimal HITS cutoff score of 6 was $78 \%$ (95\% CI $64 \%$ to $88 \%$ ), specificity $80 \%$ (95\% CI $71 \%$ to $87 \%$ ), positive likelihood ratio 3.9 (95 \% CI 2.61 to 5.76), negative likelihood ratio 0.27 (95\% CI 0.16 to 0.47 ), positive predictive value 0.61 (95\% CI $0.47,0.73$ ), and negative predictive value 0.90 (95\% CI 0.82, 0.95).

CONCLUSIONS: For a low-burden screen, the HITS demonstrated good accuracy in detecting past-year IPV relative to the CTS- 2 in a sample of female VHA patients with an optimal cutpoint of 6 . The HITS may help VHA and other health-care providers detect past-year IPV and deliver appropriate care for female Veterans.

Received January 25, 2013

Revised March 16, 2013

Accepted June 6, 2013

Published online July 4, 2013
KEY WORDS: diagnostic accuracy; sensitivity and specificity; partner abuse; screening; women veterans.

J Gen Intern Med 28(10):1288-93

DOI: $10.1007 / \mathrm{s} 11606-013-2534-x$

(c) Society of General Internal Medicine 2013

I ntimate partner violence (IPV) refers to actual or threatened physical, sexual, and psychological abuse by a current or former intimate partner. ${ }^{1}$ IPV is a major public health problem that causes and exacerbates physical health problems, including cardiovascular, respiratory, chronic pain, gynecological disorders, and adverse pregnancy outcomes. $^{2-4}$ Similarly, IPV increases risk for psychiatric conditions such as posttraumatic stress disorder (PTSD), other anxiety disorders, depression, substance misuse, eating disorders, and suicidality. ${ }^{3-5}$

Relative to civilians, female veterans are at higher risk for physical and sexual forms of IPV (33.0 \% vs. $23.8 \%$ lifetime prevalence). ${ }^{2}$ Although the reasons for elevated risk among female Veterans remain unknown, some theorize that women's exposure to violence-prone environments, such as violent childhood homes and the military, may elevate women's risk for IPV. ${ }^{6,7}$ IPV may be particularly common among women seeking care at Veterans Health Affairs (VHA) health-care facilities. ${ }^{6,8,9}$ Studies have found lifetime reports of IPV as high as $74 \%$ among women receiving medical or mental health care at a specialty VHA women's health clinic, ${ }^{6}$ and reports of past-year IPV among $24 \%$ of women under 50 years old at one large Midwestern facility. ${ }^{9}$ VHA is the largest single provider of health care for female veterans, ${ }^{10}$ indicating a critical need to identify brief and sensitive screens for identifying IPV among this at-risk population.

IPV screening programs can have benefits for women's health and safety, ${ }^{11}$ but no extant IPV screening tool has yet been rigorously evaluated in a published study of female VHA patients. It is important to establish the validity of an IPV screening tool with female VHA patients prior to implementation of such a tool in the VHA setting because 
some aspects of diagnostic accuracy, such as sensitivity and specificity, can vary by characteristics of patient populations, such as prevalence. ${ }^{12}$ The four-item Hurt/Insult/ Threaten/Scream (HITS) tool ${ }^{13}$ is one of the more efficient and sensitive validated screens in the broader literature. ${ }^{11}$ It can be administered within a couple of minutes, is easy to score, and has demonstrated sensitivity and specificity. ${ }^{14-16}$ The HITS includes items assessing psychological IPV, as well as physical aggression, a component of IPV that is lacking in other screens. ${ }^{17}$ It is critical to assess psychological IPV because it is strongly and independently associated with adverse health outcomes. ${ }^{18-20}$

In preparation for the development and implementation of national guidelines for IPV screening and responding within the VHA, we investigated the accuracy of the HITS as a predictor of past-year IPV among a sample of female VHA patients, using the Conflict Tactics Scale-Revised (CTS-2) ${ }^{21}$ as the reference standard. The CTS- 2 is the most widely used comprehensive measure of IPV in the social science and medical literature ${ }^{22}$ and is the most commonly applied reference standard for evaluating IPV screening tools. ${ }^{11,17}$ Because the HITS does not include an item assessing sexual IPV, a secondary aim of this study was to evaluate the accuracy of the HITS in detecting past-year sexual IPV.

\section{METHODS}

\section{Participants}

Women were sampled from the population of women veteran VHA patients 18 years or older in New England $(10,840$ women in fiscal year 2011). The sampling pool of 700 randomly selected women veterans was drawn from the VHA Corporate Data Warehouse, which includes names and addresses extracted from VHA electronic health records. Of this sampling pool, 581 veterans with locatable addresses received a survey and 369 participated (63.5\% response rate). Comparisons between responders and non-responders on demographic characteristics from the roster file revealed that the only difference between these groups was age, such that responders were slightly older (Cohen's $d=0.27$ ). Among responders, 179 women reported an intimate relationship within the past year; of these, the $160(89.4 \%$ of eligible women) who completed both of the IPV instruments constituted the final study sample. Women who completed only one or neither of the IPV instruments (and were excluded from the study sample) did not differ from included women on any of the sample characteristics described below (all $p$-values $>0.05$ ). There were no other exclusion criteria.

\section{Procedure}

A paper-and-pencil mail survey was administered between February and April 2012 using a modified Dillman ${ }^{23}$ multiple mailing strategy. The survey procedures were as follows: (1) informed consent fact sheet, survey packet and $\$ 10$ cash incentive were sent to all potential participants; (2) 2 weeks later a thank you/reminder postcard was sent to all potential participants; (3) 4 weeks later an additional survey packet and another $\$ 10$ cash incentive were sent to all potential participants who had not yet returned a survey or a form declining participation. Half of the survey packets presented the HITS prior to the CTS-2, and vice versa, to control for potential order effects. Potential participants were provided with the first author's phone number and other resources. This study was approved by the Institutional Review Board of the VA Boston Healthcare System.

\section{Measures}

IPV Index Test. The HITS screening tool ${ }^{13}$ comprises the following four items: "Over the last 12 months, how often did your partner: (1) physically hurt you, (2) insult you or talk down to you, (3) threaten you with harm, and (4) scream or curse at you?" Responses are scored on a 5-point scale from 1 ("never") to 5 ("frequently") and summed for a total score (range 4-20). Findings support the concurrent validity of this instrument. ${ }^{13,15,16}$ In addition, support for the reliability, sensitivity, and specificity of this instrument has been provided in several studies, although results vary with regard to the most appropriate cutoff score identified for different samples. Specifically, while findings from a sample of general female primary care patients were compared with a sample of women who were currently seeking help for IPV indicate that a cutoff score of 11 has a sensitivity of $96 \%$ and specificity of $91 \%$ in detecting IPV victims, ${ }^{13}$ other studies have supported a lower cutoff score of 6 in some diverse populations, including ethnic minority women. ${ }^{16}$

IPV Reference Standard. The CTS- $2^{21}$ is considered a "gold standard" for assessing IPV and includes 39 behaviorally specific statements that comprise five scales (physical assault, sexual coercion, psychological aggression, injury, and negotiation). ${ }^{11,17,22}$ For each statement, participants indicate how frequently the behavior happened in the past year using a 7-point scale from 0 ("never") to 6 (">20 times"). Based on expert consensus, the three subscales of the CTS-2 that are most germane to determining the presence of IPV were examined for this study: physical assault (e.g., shoving, punching, choking, and beating up), sexual coercion (e.g., being forced to do various sexual acts), and severe psychological aggression (e.g., threatening to hit or throw something and destroying things). To promote early detection, a relatively liberal criterion was used for identifying IPV to avoid "missing" exposed women. Consistent with several previous studies, ${ }^{24-26}$ IPV-positive status (yes/no) on the CTS2 was determined by a score of 1 or higher on the physical, sexual, and/or severe psychological aggression subscales, an 
approach supported by the author of the CTS-2. ${ }^{22}$ The CTS-2 has well-established psychometric properties, including good reliability as well as good construct, convergence, discriminant, and factorial validity. ${ }^{21,22,27}$

Health Symptoms. PTSD symptoms were assessed using the well-validated PTSD Checklist (PCL) ${ }^{28}$ which assesses how bothered respondents have been by the $17 D S M-I V^{29}$ PTSD symptoms over the past month on a 5-point scale from 1 ("not at all") to 5 ("extremely"). Items were summed to create a total score $(\alpha=0.97)$. Depression symptoms were measured using the 20-item Center for Epidemiologic Studies Depression Scale (CES-D) ${ }^{30}$ which assesses the past-week frequency of depression-related symptoms on a 4-point scale from 0 ("none of the time or less than 1 day") to 3 ("57 days"). Items were summed to create a total score $(\alpha=0.82)$. Current self-perceived health was assessed with the following question from the well-validated SF-36 Health Survey ${ }^{31}$ : "In general, would you say your health is excellent, very good, good, fair or poor?"

Demographic and Military-Related Characteristics. Selfreported demographic and military-related characteristics were assessed in the mail survey and included age, race/ ethnicity, highest level of education, income, military service branch, rank, and service length.

\section{Statistical Analysis}

Demographic, military, and health-related characteristics were examined by IPV status on the CTS-2, computing chi-square tests for categorical variables and t-tests for continuous variables to test for group differences. We conducted receiver-operating characteristic (ROC) curve analysis to assess the overall accuracy of the HITS, using the CTS-2 as the reference. A ROC curve displays the possible values of sensitivity (true positive rate) as a function of the false positive rate $(1-$ specificity) for different cutoff scores on the screening tool. The area under the ROC curve is the evaluative measure, which ranges from 0.5 (random performance) to 1.0 (perfect performance). For each HITS cutoff, we calculated diagnostic efficiency (overall accuracy or hit rate), kappa statistic (chance-corrected agreement with CTS-2), sensitivity (proportion of IPV-positive cases correctly detected), specificity (proportion of IPV-negative cases correctly ruled out), positive predictive value (PPV; probability that a positive screen reflects a true IPV-positive case), negative predictive value (NPV; probability that a negative screen reflects a true IPV-negative case), and positive and negative likelihood ratios (LR). The $95 \%$ confidence intervals (CIs) for proportions were calculated according to the efficient-score method. ${ }^{32}$ Finally, because the HITS does not include a sexual assault item, another ROC curve analysis was computed to determine the sensitivity and specificity of the HITS specifically in detecting sexual IPV as measured by the CTS- 2 .

\section{RESULTS}

Past-Year IPV. In this sample, 46 (28.8 \%) women reported past-year IPV on the CTS-2. Among women who experienced IPV, 23 (50.0\%) reported any physical IPV, $23(50.0 \%)$ reported any sexual IPV, and $29(63.0 \%)$ reported any severe psychological IPV. Nearly half $(47.8 \%)$ of these women reported more than one type of IPV.

Participant Characteristics by IPV Status. Table 1 displays participants' demographic, military-related characteristics, and health indicators by IPV status on the CTS-2. Women with past-year IPV were younger and were almost twice as likely to identify as a racial/ethnic minority as women without past-year IPV (30.4\% vs. $15.8 \%$ ). Women with past-year IPV served in the military for significantly less time and were more likely to have served in the Army than women without past-year IPV. There were no other group differences on demographic/military characteristics (all $p \mathrm{~s}>$ $0.05)$. For current health symptoms, women with past-year IPV reported significantly higher levels of PTSD and depression symptoms, as well as lower levels of perceived health, than women without past-year IPV.

Diagnostic Accuracy. The area under the ROC curve (Fig. 1) was 0.85 (CI: 0.78, 0.91), indicating that the HITS generally performed well in this sample. Table 2 summarizes the accuracy of the HITS at different cutoff scores relative to the CTS- 2 and demonstrates that a cutoff score of 6 optimizes efficiency (79\% accurate) with the best balance of sensitivity (78 \%) and specificity ( $80 \%)$ in this sample. At the optimal cutoff of $6,61 \%$ of positive screen results reflected true IPV-positive cases and $90 \%$ of negative screen results reflected true IPV-negative cases. Kappa agreement was 0.54 .

An additional ROC analysis was conducted to determine whether the HITS accurately detects women who have experienced past-year sexual IPV as measured on the CTS-2 (as opposed to any IPV). Findings were similar, albeit more modest, than when detecting any IPV. As shown in Figure 1, the area under the ROC curve was 0.79 (CI: 0.69, 0.89), indicating that the HITS had good agreement with the CTS-2 sexual IPV subscale. Evaluation of different cutoff points on the HITS demonstrates that a cutoff score of 6 optimizes efficiency ( $74 \%$ accurate) with the best balance of sensitivity $(87 \%)$ and specificity $(72 \%)$ in detecting past-year sexual IPV. 
Table 1. Sample Demographic, Military, and Health-Related Characteristics by IPV Status $(n=160)$

\begin{tabular}{|c|c|c|}
\hline Respondent characteristics & $\begin{array}{l}\text { IPV + Women } \\
n=46\end{array}$ & $\begin{array}{l}\text { IPV - Women } \\
n=114\end{array}$ \\
\hline Age $(\text { years, mean } \pm S D)^{*}$ & $43.78 \pm 12.71$ & $49.25 \pm 14.49$ \\
\hline \multicolumn{3}{|l|}{ Race, $n(\%)^{*}$} \\
\hline White & $32(69.6)$ & $96(84.2)$ \\
\hline Non-White & $14(30.4)$ & $18(15.8)$ \\
\hline \multicolumn{3}{|l|}{ Education, $n(\%)$} \\
\hline $\begin{array}{l}\text { High school, GED, vocational } \\
\text { or technical training }\end{array}$ & $7(15.2)$ & $20(17.5)$ \\
\hline Some college & $27(58.7)$ & $42(36.8)$ \\
\hline $\begin{array}{l}\text { 4-year college graduate } \\
\text { or beyond }\end{array}$ & $12(26.1)$ & $51(44.7)$ \\
\hline Missing & $0(0)$ & $1(1.0)$ \\
\hline \multicolumn{3}{|l|}{ Household income, $n(\%)$} \\
\hline$\$ 15,000$ or less & $4(8.7)$ & $9(7.9)$ \\
\hline$\$ 15,001-\$ 25,000$ & $8(17.4)$ & $14(12.3)$ \\
\hline$\$ 25,001-\$ 35,000$ & 9 (19.6) & $15(13.2)$ \\
\hline$\$ 35,001-\$ 50,000$ & 9 (19.6) & $21(18.4)$ \\
\hline$\$ 50,001-\$ 75,000$ & $6(13.0)$ & $22(19.3)$ \\
\hline$\$ 75,001-\$ 100,000$ & $3(6.5)$ & $14(12.3)$ \\
\hline$\$ 100,000$ or more & $5(10.9)$ & $8(7.0)$ \\
\hline Missing & $2(4.3)$ & $11(9.6)$ \\
\hline \multicolumn{3}{|l|}{ Marital status, $n(\%)$} \\
\hline Married & $27(59.0)$ & $65(57.0)$ \\
\hline Unmarried & $19(41.0)$ & $49(43.0)$ \\
\hline $\begin{array}{l}\text { Length of military service } \\
\text { (years, mean } \pm S D)^{*}\end{array}$ & $2.64 \pm 0.92$ & $3.51 \pm 1.06$ \\
\hline \multicolumn{3}{|l|}{ Branch of service, $n(\%)^{*}$} \\
\hline Army & $30(65.2)$ & $48(42.1)$ \\
\hline Navy & $4(8.7)$ & $34(29.8)$ \\
\hline Air Force & $8(17.4)$ & $24(21.1)$ \\
\hline Marines & $2(4.3)$ & $4(3.5)$ \\
\hline Coast Guard & $1(2.2)$ & $1(0.9)$ \\
\hline Missing & $1(2.2)$ & $3(2.6)$ \\
\hline \multicolumn{3}{|l|}{ Rank, $n(\%)$} \\
\hline Enlisted & $40(87.0)$ & $97(85.1)$ \\
\hline Officer/Warrant Officer & $5(10.9)$ & $12(10.5)$ \\
\hline Missing & $1(2.2)$ & $5(4.4)$ \\
\hline \multicolumn{3}{|l|}{ Health indicators (mean $\pm S D$ ) } \\
\hline PTSD* & $46.0 \pm 19.42$ & $33.8 \pm 16.71$ \\
\hline Depression* & $25.2 \pm 11.71$ & $20.2 \pm 8.42$ \\
\hline Self-perceived health* & $2.82 \pm 0.96$ & $3.20 \pm 0.96$ \\
\hline
\end{tabular}

Percentages may not equate to $100 \%$ because of rounding and/or missing data for participant characteristics. Due to missing data, $n$ varied from 140 to 160 . *Denotes a significant difference between groups, $p<0.05$. Self-perceived health ranges from 1(poor) to 5 (excellent), with higher scores reflecting higher perceived health

\section{DISCUSSION}

The current findings are promising with respect to the clinical utility of the HITS with female VHA patients. Findings indicated that a cutoff score of 6 on the four-item tool accurately detected $78 \%$ of women identified as abused within the past year by the more comprehensive and behaviorally specific CTS-2. This cutoff score is lower than that suggested by the original validation work on this tool. This is perhaps not surprising given that the original validation study was based on a comparison of a sample of help-seeking IPV victims to a general sample of female primary care patients. ${ }^{13}$ In contrast, the current study focused on general female VHA patients with the goal of promoting early detection of IPV, including cases of severe psychological IPV in the absence of recent physical or

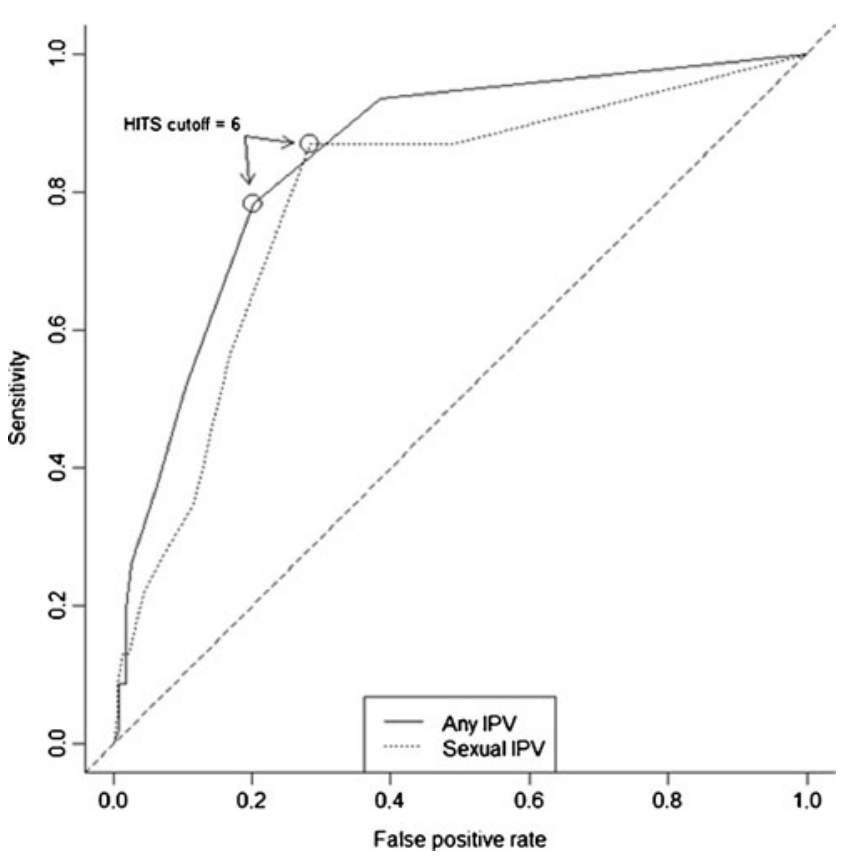

Figure 1. Receiver-operating characteristic (ROC) curve of the HITS $(N=160)$ for detecting any IPV (solid line) and sexual IPV (dotted line) as measured by the CTS-2. For any IPV, the area under the curve $=0.85, S E=0.03$, CI: $0.78,0.91$. For sexual IPV, the area under the curve $=0.79, S E=0.05$, CI: $0.69,0.89$.

sexual IPV. Presuming a fairly representative prevalence of IPV, the majority of positive screens at this cutoff score (61\%) should reveal true cases of IPV, whereas IPV can be ruled out with confidence for the great majority of negative screens $(90 \%)$. In order to improve the $61 \%$ PPV (i.e., by decreasing false positives) by means of increasing the cutoff score, sensitivity would be unacceptably low $(52 \%$ at a cutoff score of 7). Conversely, adopting a cutoff score of 5 to improve sensitivity, the validity of positive screens would fall to chance level (49\% PPV). Thus, the cutoff score of 6 offers a balance between moderately good sensitivity and a tolerable number of false-positive screens. Given the significant adverse health risks associated with IPV, ${ }^{1}$ the potential benefits of identification for women's health and safety ${ }^{11}$ likely outweigh the relatively modest costs associated with false-positive cases (i.e., provider time for follow-up).

Nearly $29 \%$ of women in this study reported past-year physical, sexual, and/or severe psychological IPV. This finding is in the upper range of what has been found among other samples of female health-care patients, ${ }^{11}$ providing additional support for the finding that IPV is a pervasive health issue among female veterans. Findings suggest that subgroups of female veterans may be at higher risk for pastyear IPV, including women who served in the military for less time and those who served in the Army. Such factors should be further examined in future research. As expected, past-year IPV was associated with significantly higher levels of distress, including elevated symptoms of PTSD and depression, relative to veterans who did not report past-year IPV. These 
Table 2. Operating Characteristics of the HITS in Detecting Past-Year IPV at Different Cutoff Scores $(N=160)$

\begin{tabular}{|c|c|c|c|c|c|c|c|c|}
\hline $\begin{array}{l}\text { HITS } \\
\text { score }\end{array}$ & $\begin{array}{l}\text { Kappa } \\
(S E)\end{array}$ & Efficiency & $\begin{array}{l}\text { Sensitivity } \\
(95 \% \text { CI) }\end{array}$ & $\begin{array}{l}\text { Specificity } \\
(95 \% \text { CI) }\end{array}$ & LR+ (95 \% CI) & LR- (95\% CI $)$ & PPV (95 \% CI) & NPV $(95 \%$ CI $)$ \\
\hline$\geq 5$ & $0.43(0.06)$ & 0.71 & $0.94(0.81,0.98)$ & $0.61(0.52,0.70)$ & $2.42(1.90,3.09)$ & $0.11(0.04,0.32)$ & $0.49(0.39,0.60)$ & $0.96(0.88,0.99)$ \\
\hline$\geq 6$ & $0.54(0.07)$ & 0.79 & $0.78(0.63,0.89)$ & $0.80(0.71,0.87)$ & $3.88(2.61,5.76)$ & $0.27(0.16,0.47)$ & $0.61(0.47,0.73)$ & $0.90(0.82,0.95)$ \\
\hline$\geq 7$ & $0.45(0.08)$ & 0.79 & $0.52(0.37,0.67)$ & $0.89(0.82,0.94)$ & $4.96(2.71,9.05)$ & $0.53(0.39,0.72)$ & $0.67(0.49,0.81)$ & $0.82(0.74,0.88)$ \\
\hline$\geq 8$ & $0.36(0.08)$ & 0.78 & $0.37(0.24,0.52)$ & $0.94(0.87,0.97)$ & $6.02(2.67,13.50)$ & $0.67(0.54,0.84)$ & $0.71(0.49,0.87)$ & $0.79(0.71,0.85)$ \\
\hline$\geq 9$ & $0.29(0.08)$ & 0.77 & $0.26(0.15,0.14)$ & $0.97(0.92,0.99)$ & $9.91(2.93,33.51)$ & $0.76(0.64,0.90)$ & $0.80(0.51,0.95)$ & $0.77(0.69,0.83)$ \\
\hline$\geq 10$ & $0.23(0.07)$ & 0.76 & $0.20(0.10,0.34)$ & $0.98(0.93,0.99)$ & $11.15(2.50,49.65)$ & $0.82(0.71,0.94)$ & $0.82(0.48,0.97)$ & $0.75(0.67,0.82)$ \\
\hline$\geq 11$ & $0.09(0.06)$ & 0.73 & $0.09(0.03,0.22)$ & $0.98(0.93,0.99)$ & $4.96(0.94,26.13)$ & $0.93(0.85,1.01)$ & $0.67(0.24,0.94)$ & $0.73(0.65,0.79)$ \\
\hline
\end{tabular}

SE standard error; CI confidence interval, LR likelihood ratio, PPV positive predictive value, NPV negative predictive value

findings further highlight the importance of detecting IPV because these mental health problems are often chronic in this population, ${ }^{33,34}$ and treatment of these symptoms may reduce women's risk for subsequent IPV. ${ }^{35}$ Thus, detection is critical because it provides the opportunity to treat associated health problems and offer appropriate social and mental health interventions to increase safety and well-being.

This study extends previous work on the HITS by examining its utility in detecting sexual IPV. Although the original HITS does not include a sexual aggression item, findings suggest that the HITS performs fairly well in identifying cases of sexual IPV in this sample because women who report sexual IPV also often report physical and psychological IPV ${ }^{18,19}$. Consistent with findings for the cutoff score for overall IPV in this sample, a cutoff score of 6 maximized sensitivity and specificity in detecting sexual IPV. In fact, a cutoff score of 6 resulted in a sensitivity of $87 \%$. Thus, cases of sexual IPV do not go undetected or "missed" at any higher rates than physical and severe psychological IPV.

This study has limitations that can be addressed in future research. This study involved a mail survey. Though a strength of this methodology is that some individuals may feel more comfortable disclosing IPV experiences in a confidential survey (compared to a face-to-face interview), interviews allow respondents to clarify response options if questions arise and empathetic interviewers may experience higher rates of disclosure; thus, results may vary with the assessment context. $^{12}$ Additional research is needed to evaluate the performance of the HITS in the clinical setting and compare self-report methods (e.g., paper and pencil, computers, tablets) to clinician-administered questioning. Additionally, study participants were limited to female VHA patients in the New England geographic area, and as such, caution should be used when generalizing to national populations of VHA patients or to female veterans who are not recent VHA patients. Future research with nationally representative samples is needed to further evaluate the clinical utility of the HITS in this understudied population. It is also important to acknowledge that there is not a perfect reference standard against which to compare the HITS and other measures (e.g., Index of Spouse Abuse and Composite Abuse Scale) have served as reference standards in the broader IPV screening literature. ${ }^{11,17} \mathrm{We}$ carefully selected the CTS-2 over other available measures because it is the most widely used, psychometrically sound, comprehensive, and behaviorally specific assessment of the presence of physical, sexual, and psychological IPV. ${ }^{21,22}$ Finally, although the HITS demonstrated reasonable operating characteristics in this study, performance (e.g., sensitivity) of this tool could be improved. For example, modifying the tool to include more behaviorally specific items and response options may improve accuracy without adding significant administrative burden. Finally, future work should examine the associations between HITS responses and health indicators (e.g., PTSD diagnoses, service-connected disabilities, and frequency of health-care use) from VHA electronic health records.

The HITS has the potential to effectively and efficiently identify a high proportion of female VHA patients who have experienced past-year IPV. Given the high prevalence of past-year IPV reported in this sample and the ease of administration and interpretation (i.e., it takes only a couple of minutes for patients to complete), providers should consider using the HITS to inquire about IPV among female veterans at least annually. One approach that may make screening more standardized and acceptable for VHA providers would be to embed the HITS within the electronic medical record template as a clinical reminder ${ }^{36}$. Use of electronic medical records to facilitate IPV screening is promising because scoring can occur automatically and prompt providers to engage in specific follow-up care (e.g., provide risk assessment and the domestic violence hotline number, request contract information for a safe relative or friend who may know the patients' contact information in the event that patient has to move or change phone numbers, and place referrals). As argued elsewhere, ${ }^{36,37}$ successful IPV screening within VHA must include mechanisms for appropriate and comprehensive responses to positive screens, including validation, education, and risk assessment, as well as coordination of primary care, mental health, social work, legal services, and victim advocacy both within the VHA and in the community.

Acknowledgments: The authors wish to thank Drs. Errol Baker, Mark Meterko, and Paula Schnurr for consultation on the methods and data analytic strategies utilized in this manuscript. The authors 
also express their gratitude to Jason Atkins, RN-BC, MBI, Chief of Health Informatics for the New England Veterans Integrated Service Network, for his consultation on and provision of roster data.

This research was supported by the Department of Veterans Affairs, Veterans Health Administration, Health Services Research and Development (HSR\&D) as part of Dr. Katherine Iverson's HSR\&D Career Development Award (CDA 10-029). The views expressed in this article are those of the authors and do not necessarily reflect the position or policy of the Department of Veterans Affairs or the US government.

Conflict of Interest: The authors declare that they do not have any conflicts of interest.

Funding Support: This research was supported by the Department of Veterans Affairs, Veterans Health Administration, Health Services Research and Development (HSR\&D) Services as part of Dr. Katherine Iverson's HSR\&D Career Development Award (CDA 10-029). The views expressed in this article are those of the authors and do not necessarily reflect the position or policy of the Department of Veterans Affairs or the US government.

Corresponding Author: Katherine M. Iverson, $\mathrm{PhD}$; Women's Health Sciences Division of the National Center for PTSD, VA Boston Healthcare System, 150 South Huntington Avenue (116B-3), Boston, MA 02130, USA (e-mail: Katherine.Iverson@va.gov).

\section{REFERENCES}

1. Centers for Disease Control. Understanding intimate partner violence. 2012; http://www.cdc.gov/violenceprevention/pdf/ipv_factsheet-a.pdf. Accessed 05-30-2013.

2. Dichter ME, Cerulli C, Bossarte RM. Intimate partner violence victimization among women veterans and associated heart health risks. Womens Health Issues. 2011;21(4):S190-4.

3. Campbell JC. Health consequences of intimate partner violence. Lancet. 2002;359(9314):1331-6.

4. Humphreys J, Cooper BA, Miaskowski C. Occurrence, characteristics, and impact of chronic pain in formerly abused women. Violence Against Women. 2011;17(10):1327-43.

5. Iverson KM, Dick A, McLaughlin KA, et al. Exposure to interpersonal violence and its associations with psychiatric morbidity in a US national sample: a gender comparison [published online ahead of print December 17, 2012]. Psychol Violence.

6. Campbell R, Greeson MR, Bybee D, Raja S. The co-occurrence of childhood sexual abuse, adult sexual assault, intimate partner violence, and sexual harassment: a mediational model of posttraumatic stress disorder and physical health outcomes. J Consult Clin Psychol. 2008;76(2):194-207.

7. Sadler AG, Booth BM, Mengeling MA, Doebbeling BN. Life span and repeated violence against women during military service: effects on health status and outpatient utilization. J Womens Health. 2004; 13(7):799-811.

8. Caralis PV, Musialowski R. Women's experiences with domestic violence and their attitudes and expectation regarding medical care of abuse victims. South Med J. 1997;90(11).

9. Murdoch M, Nichol KL. Women veterans' experiences with domestic violence and with sexual harassment while in the military. Arch Fam Med. 1995;4(5):411-8.

10. Frayne SM, Phibbs CS, Friedman SA, et al. Sourcebook: Women Veterans in the Veterans Health Administration. Volume 1. Sociodemographic Characteristics and Use of VHA Care. Washington, DC: Women's Health Evaluation Initiative, Women Veterans Health Strategic Health Care Group, Veterans Health Administration, Department of Veterans Affairs; 2010.

11. Nelson HD, Bougatsos C, Blazina I. Screening women for intimate partner violence: a systematic review to update the US Preventive Services Task Force recommendation. Ann Intern Med. 2012;156(11):796-808.

12. Bossuyt PM, Reitsma JB, Bruns DE, et al. The STARD statement for reporting studies of diagnostic accuracy: explanation and elaboration. Clin Chem. 2003;49(1):7-18.
13. Sherin KM, Sinacore JM, Li XQ, Zitter RE, Shakil A. HITS: a short domestic violence screening tool for use in a family practice setting. Fam Med. 1998;30:508-12.

14. Chan C, Chan Y, Au A, Cheung G. Reliability and validity of the "Extended - Hurt, Insult, Threaten, Scream" (E-HITS) screening tool in detecting intimate partner violence in hospital emergency departments in Hong Kong. Hong Kong J Emerg Med. 2010;17(2):109-17.

15. Chen PHRS, Washington J, Jacobs A, Vega M, Pan KY, Johnson MS. Randomized comparison of 3 methods to screen for domestic violence in family practice. Ann Fam Med. 2007;5(5):430-5.

16. Chen P-H, Rovi S, Vega M, Jacobs A, Johnson MS. Screening for domestic violence in a predominantly Hispanic clinical setting. Fam Pract. 2005;22(6):617-23.

17. Rabin RF, Jennings JM, Campbell JC, Bair-Merritt MH. Intimate partner violence screening tools: a systematic review. Am J Prev Med. 2009;36(5):439-45.

18. Coker AL, Davis KE, Arias I, et al. Physical and mental health effects of intimate partner violence for men and women. Am J Prev Med. 2002;23(4):260-8.

19. Pico-Alfonso MA. Psychological intimate partner violence: the major predictor of posttraumatic stress disorder in abused women. Neurosci Biobehav Rev. 2005;29(1):181-93.

20. Pico-Alfonso MA, Garcia-Linares MI, Celda-Navarro N, Blasco-Ros C, Echeburua E, Martinez M. The impact of physical, psychological, and sexual intimate male partner violence on women's mental health: depressive symptoms, posttraumatic stress disorder, state anxiety, and suicide. J Women's Health. 2006;15:599-611.

21. Straus MA, Hamby SL, Boney-McCoy S, Sugarman DB. The revised conflict tactics scale (CTS2): development and preliminary psychometric data. J Fam Issues. 1996; 17:283-316.

22. Straus MA. Conflict tactics scales. In: Jackson NA, ed. Encyclopedia of Domestic Violence. New York: Routledge: Taylor \& Francis Group; 2007:190-7.

23. Dillman DA. Mail and Internet Surveys: The Tailored Design Method. 2, Illustrated. ed. New Jersey: John Wiley and Sons; 2007.

24. Dubowitz H, Prescott L, Feigelman S, Lane W, Kim J. Screening for intimate partner violence in a pediatric primary care clinic. Pediatrics. 2008; 121(1):e85-91.

25. Houry D, Feldhaus K, Peery B, et al. A positive domestic violence screen predicts future domestic violence. J Interpers Violence. 2004; 19(9):955-66.

26. Koziol-McLain J, Coates CJ, Lowenstein SR. Predictive validity of a screen for partner violence against women. Am J Prev Med. 2001;21(2):93-100.

27. Straus H, Cerulli C, McNutt LA, et al. Intimate partner violence and functional health status: associations with severity, danger, and selfadvocacy behaviors. J Women's Health (Larchmt). 2009; 18(5):625-31.

28. Weathers F, Litz BT, Herman DS, Huska JA, Keane TM. The PTSD Checklist (PCL): Reliability, Validity, and Diagnostic Utility. Paper presented at: Presentation at the International Society for Traumatic Stress Studies 1993; San Antonio, TX.

29. APA. Diagnostic and Statistical Manual of Mental Disorders. 4th ed. Washington, D.C: American Psychological Association; 2000.

30. Radloff LS. The CES-D scale: a self-report depression scale for research in the general population. Appl Psychol Meas. 1977;1(3):385-401.

31. Ware JE. SF-36 Health Survey: Manual and Interpretation Guide. Boston: The Health Institute, New England Medical Center; 1993.

32. Newcombe RG. Two-sided confidence intervals for the single proportion: comparison of seven methods. Stat Med. 1998;17(8):857-72.

33. Campbell JC, Soeken KL. Women's responses to battering over time: ananalysis of change. Journal of Interpersonal Violence. 1999;14:21-40.

34. Zlotnick C, Johnson DM, Kohn RM. Intimate partner violence and long-term psychosocial functioning in a national sample of American women. Journal of Interpersonal Violence. 2006;21(2):262-75.

35. Iverson KM, Gradus JL, Resick PA, Suvak MK, Smith KF, Monson CM. Cognitive-behavioral therapy for PTSD and depression symptoms reduces risk for future intimate partner violence among interpersonal trauma survivors. J Consult Clin Psychol. 2011;79(2):193-202.

36. Iverson KM, Well SY, Wiltsey-Stirman S, Vaughn R, Gerber MR. VHA primary care providers' perspectives on screening female Veterans for intimate partner violence: a preliminary assessment. J Family Violence. In press.

37. Sweeney AC, Weitlauf JC, Manning EA, Sze JA, Waldrop AE, Hasser C. Intimate partner violence: perspectives on universal screening for women in VHA primary care. Women's Health Issues. 2013;23(2):e73-6. 